Article

\title{
Possible correlation of electrochemiluminescence based numerical cut off index value with concentration of anti-SARS-CoV-2 antibody: Is it worth reporting?
}

\author{
Shabnum Khawaja, ${ }^{1}$ Mohammad Asif, ${ }^{2}$ Samina Naz Mukry, ${ }^{2}$ Tahir Sultan Shamsi ${ }^{2}$ \\ ${ }^{1}$ Department of Chemical Pathology; ${ }^{2}$ Department of Haematology, National Institute of Blood disease and Bone \\ Marrow Transplantation (NIBD), Karachi, Pakistan
}

\begin{abstract}
Background: Many laboratories are reporting a numerical cutoff index value (COI) value for most anti-SARS-CoV-2 qualitative tests. These numerical values in patients' report ultimately created great confusion in the public and physicians, therefore this study was designed to evaluate the correlation of electrochemiluminescence (ECLIA) based numerical COI values with quantitative ELISA of anti-SARS-CoV-2 antibody.

Design and Methods: Two hundred and twenty-eight (228) recovered COVID-19 patients were included; their serum samples were analyzed by quantitative ELISA and ECLIA for anti-SARSCOV-2 antibodies.

Results: One hundred and seventy-three (75.8\%) patients tested positive by ECLIA and ELISA assay and thirty-seven (6.2\%) were tested negative by both methods. A weak positive correlation $(r=0.37)$ was found between numerical COI value of ECLIA with ELISA concentration, which was statistically significant with $\mathrm{p}<0.001$. All values were dispersed on scatter plot and there was no significant linear relationship between ECLIA and ELISA assay.

Conclusions: As both testing techniques are base upon the same immunological phenomena of detecting antibodies against nucleocapsid protein. We suggest that COI values are not meant to describe the immunity level of the individuals thus the physicians should not consider it as a quantitative value for antibody levels in COVID-19 patients.
\end{abstract}

\section{Introduction}

On March 11, 2020 the World Health Organization (WHO) declared COVID-19 a global pandemic. ${ }^{1}$ The causative agent of COVID-19 is a corona virus named as SARS-CoV-2 and it belongs to a family of viruses that may cause respiratory symptoms ranging from common cold to severe pneumonia. The host or infected individual in response to the infection produces specific antibodies including IgM, IgG and IgA. ${ }^{2,3}$ The detection of these antibodies indicates that the individual has been exposed to the virus. During the recent pandemic, several diagnostics companies introduced testing kits for detection of these antibodies, to estimate the percentage of the population previously infected with the virus information needed to devise strategies for community surveillance in order to protect the public's health. ${ }^{4}$ Many tests based on different techniques are readily available in the market to detect the antibody. The two most commonly used techniques to detect antibodies against SARS$\mathrm{CoV}-2$ that entered the market included Electrochemiluminescence Immunoassay (ECLIA) and Enzyme-linked Immunosorbent assay (ELISA). ECLIA is a qualitative assay used to find the patients who had been exposed to the virus while utilizing cut off index (COI) as a reference to give a positive or negative result. The test targets antibodies including IgG, IgM and IgA against nucleocapsid of SARS$\mathrm{CoV}-2$. On the other hand, ELISA is a quantitative test intended to define the titers of $\operatorname{IgG}$ in patients who had been previously exposed. ${ }^{5}$ In case of SARS-CoV-2, it is still unknown if the presence of IgG confers any protection against re-infection. As the number of COVID cases increased, there was emergence of different techniques to estimate the exposure of the population to the causative agent and to detect the development of corresponding antibodies. New techniques were developed and marketed to diagnose the disease in acute phase as well as to show the percentage of the population that had been exposed to the virus. ${ }^{6}$ On the other hand, there were techniques which were intended to show the quantitative values of one specific types of antibody. Of these testing systems, ECLIA based testing system and ELISA based Testing system have been used by many laboratories in Pakistan. ${ }^{7}$

Both testing systems have been claimed to have high sensitivity and specificity, ${ }^{8}$ but COI is mistakenly considered as a quantitative variable to define the overall anti- SARS-CoV-2 antibodies in recovered patients. The objective of the current study was to record any possible correlation of ECLIA based numerical COI values provided by Roche Diagnostics (Rotkreuz, Switzerland). testing system with quantitative ELISA provided by AEKULISA ${ }^{\circledR}$ (AESKU Diagnostics; Wendelsheim, Germany) for anti- SARSCoV-2 antibody detection.

Significance for public health

Many laboratories in Pakistan are reporting numerical COI values, which ultimately created great confusion among the patients and physicians. These values are, used indiscriminately and wrongly compared to other testing systems which were in general intended to be used for quantitative analysis of the antibodies developed in the persons exposed to the virus. There had been obvious misunderstanding in the public including the healthcare sector when these different techniques were used indiscriminately without a proper orientation towards the utility and limitations of a given testing system. As a result, the different numerical COI values which were included in the laboratory reports of the test created a great havoc and raised suspicions about the certainty of the diagnostic techniques. This correlation is important because this number game has been talk of the town and lay person uses them to get the idea of one's own immunity status. 


\section{Design and Methods}

This prospective study was performed from $5^{\text {th }}$ June to $30^{\text {th }}$ October 2020 at National Institute of Blood Disease and Bone Marrow Transplantation (NIBD) Hospital, Karachi, Pakistan. The adult fully recovered corona survivors of either gender aged 18 to 60 years were recruited after two weeks of negative PCR. All subjects were healthy with no known co-morbidities. The study was approved by institution's ethical review committee. Three to five $\mathrm{ml}$ blood sample was taken in EDTA tube for anti-SARS-CoV-2 antibody analysis by both ELISA and ECLIA. For quantitative ELISA AESKULISA ${ }^{\circledR}$ SARS-CoV-2 NP IgG (AESKU Diagnostics) was used the results were interpreted as positive at cutoff $>12 \mathrm{U} / \mathrm{ml}$, while $<12 \mathrm{U} / \mathrm{ml}$, were labeled as negative. Total antibody $\operatorname{IgG}, \operatorname{IgM}$ and $\operatorname{IgA}$ done by ECLIA on Cobas e-411 Immunoassay analyzer by Roche Diagnostics Cutoff index $\geq 1.0$ used to label as reactive, while $\leq 1.0$ used to label as non-reactive as per manufacturer recommendations.

\section{Statistical analysis}

Data was analyzed by EP evaluator (vers. 10.0). Mean and Standard deviation for quantitative variables were calculated along with frequencies for all categorical variables. The mean values for ECLIA anti-SARS-CoV-2 were calculated by using numerical cut of index (COI) value. Scatter plots was created by using test method as ECLIA on Y-axis and reference method as ELISA on Xaxis to see the relationship between COI and concentration. Correlation coefficient ( $\mathrm{r}$ value) was calculated by Pearson correlation. Cohen's Kappa was calculated to see the agreement, Cohen's Kappa $>75 \%$ considered as high agreement. Furthermore, $t$-test was applied to compare the means of ECLIA and ELISA and $p$ value of less than 0.05 was taken as statistically significant.

\section{Results}

Two hundred and twenty-eight (228) COVID-19 survivors were included in the study. The mean age of subjects was $36.6 \pm 11.6$ years. There were $192(84.2 \%)$ males and $62(15.8 \%)$ females. The mean anti-SARS-CoV-2 antibody by ECLIA was $30.38 \pm 31.04 \mathrm{COI}$ and by ELISA was $44.35 \pm 38.49 \mathrm{U} / \mathrm{ml}$. The mean anti-SARS-CoV-2 antibody level was higher by ELISA and there was a significant statistical difference between these two means with $\mathrm{p}$ value $<0.000$. Approximately, $75.8 \%$ (173) individuals tested positive either by ECLIA and ELISA or both whereas $16.2 \%$ (37) tested negative by both methods. About $8.34 \%$ (18) individuals tested positive by ECLIA and tested negative by ELISA assay. Antibody results obtained by ECLIA and ELISA of all patients were used to create scatter plot, which gives slope of $0.69(95 \% \mathrm{CI}$ - 0.60-0.78), intercept of -0.53 (95\% CI - 5.83-4.78) and did not show any significant linear relationship between ECLIA and ELISA. Initial values we found a slightly uphill with a positive slope but as we move forward, the data values were dispersed as seen in Figure 1. There was very little positive relationship found between the numerical COI value of ECLIA with quantitative ELISA for anti-SARS-CoV-2 antibody with correlation coefficient $\mathrm{r}=0.37$ with $\mathrm{p}$ value $<0.001$.

\section{Discussion}

The current study was inspired by the curiosity to understand the numerical COI values given by any ECLIA testing system to address the confusion existent in the public and physicians that whether higher COI values meant a higher humoral immunity level (Ig level) or not. Although manufacturer of ECLIA had clearly described the qualitative nature of the testing system, but the included numerical values or COI reporting by laboratories were creating confusion. Physicians were confused as well and were considering these COI values comparable to Ig levels reported by quantitative ELISAs. Every participant of the study was tested by two antibody-based testing systems i.e. Eclesys ${ }^{\circledR}$ Roche testing system using ECLIA technique and Quantitative ELISA by AESKULISA $^{\circledR}$ diagnostics testing system. We found lack of any significant quantitative correlation between these two. The results suggest that the COI values are not meant to describe the immunity level of the individuals. The correlation of electrochemiluminescence based numerical COI of anti-SARS-CoV-2 antibody with actual concentration is not reported in literature.

Many laboratories in Pakistan are reporting numerical COI values, which ultimately created great confusion among the patients and physicians. These values are, used indiscriminately and wrongly compared to other testing systems which were in general intended to be used for quantitative analysis of the antibodies developed in the persons exposed to the virus.

Stites et al. in 2020 determined IgG levels by using ELISA technique as the serum was collected after 28 days of infection, they did not perform IgM levels on ELISA as they collected samples after 4 weeks of illness and its already known that IgM levels markedly reduced after 4 weeks of infection. ${ }^{9}$ The positivity rate of IgM antibody was only $60 \%$, with a marked reduction in antibody levels 4 weeks after onset of illness. ${ }^{10}$ Anti-SARS-CoV-2 S-specific IgG antibodies were identifiable from day 7 onwards, peaking at approximately day 25 Serum IgG antibodies were still maintained at a high level after 4 weeks of infection. ${ }^{11}$ Hou et al also reported IgM levels increased during the first week after SARS $\square \mathrm{CoV} \square 2$ infection, peaked 2 weeks and then reduced to near $\square$ background levels in most patients. ${ }^{12}$ There were $8.34 \%$ individuals who were tested positive only by ECLIA system and negative by ELISA based system, which may be due to IgM detected by Roche and not

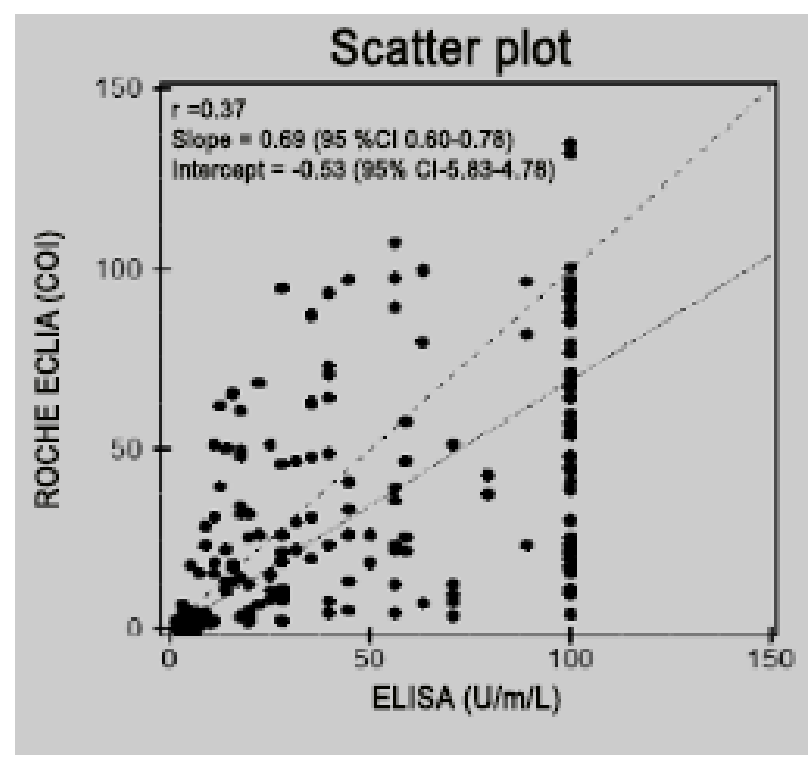

Figure 1. Scatter plot of ECLIA and ELISA assay showing weak correlation between the two assays. 
detected by ELISA although this could not be proven because of the combo nature of the Roche ECLIA testing technique. We strongly suggested that ECLIA being a qualititative test should be reported as positive or negative. No numerical values for qualitative assays should be documented in lab reports to avoid confusion in physicians as well as nonclinical individuals. This suggestion is in line with the manufacturer's claim whose intention was to give only the qualitative results, but confusion was created because of the associated numerical values. As these COI values lack any relationship to the antibody/immunity status of an individual. This practice has been adopted by our laboratory although some other laboratories in our country are still issuing reports with numerical values included thus creating confusion patients and physicians.

\section{Conclusions}

The results of this study suggest a lack of significant quantitative correlation between these two testing systems. Although greater sample size may be required to find any possible correlation, but it can be strongly suggested comparing these two systems. Additionally studies are required to explain the meaning of numerical values generated by the Roche system and their correlation with the immunity status/antibody titers in a given individual.

Correspondence: Shabnum Khawaja, Consultant Chemical Pathologist, Department of Chemical Pathology, National Institute of Blood disease and Bone Marrow Transplantation (NIBD), ST 2/A Block 17 Gulshan-e-Iqbal KDA Scheme 24, Karachi 74800, Pakistan. Fax:+92.21.4821504. E-mail: shabnam.dildar@yahoo.com

Key words: ECLIA; cutoff index values; quantitative ELISA; correlation.

Dedication: To my Mother (Late Zarina Ramzan Ali), Father (Late Ramzan Ali) and Brother (Late Rehman Ali).

Contributions: All the authors made a substantive intellectual contribution, performed part of the experiments. All the authors have read and approved the final version of the manuscript and agreed to be accountable for all aspects of the work.

Conflict of interest: The authors declare that they have no competing interests, and all authors confirm accuracy.

Ethics approval and consent to participate: This study was done after approval of Institutional Review Board (IRB)/Ethical Committee of NIBD Hospital. Written informed consent taken by each study participant.

Availability of data and materials: All data generated or analyzed during this study are included in this published article.

Received for publication: 30 November 2020.

Accepted for publication: 4 February 2021.

o Copyright: the Author(s), 2021

Licensee PAGEPress, Italy

Journal of Public Health Research 2021;10:2079

doi:10.4081/jphr.2021.2079

This work is licensed under a Creative Commons Attribution

NonCommercial 4.0 License (CC BY-NC 4.0).

\section{References}

1. WHO. General's opening remarks at the media briefing on COVID-19 - 11 March 2020. World Health Organization, 2020. Accessed: 11 Sep 2020. Available from: https://www.who.int/dg/ speeches/detail/who-director-generals-opening-remarks-at-the-media-briefing-on-covid-19---11march-2020

2. Long QX, Tang XJ, Shi QL, et al. Clinical and immunological assessment of asymptomatic SARS-CoV-2 infections. Nat Med 2020;26:1200-4.

3. European Centre for Disease Prevention and Control. Immune responses and immunity to SARS-CoV-2. 2020. Accessed: 28 Sep 2020. Available from: https://www.ecdc. europa.eu/en/ covid-19/latest-evidence/immune-responses

4. Hick JL, Hanfling D, Wynia MK, et al. Duty to plan: health care, crisis standards of care, and novel coronavirus SARSCoV-2. National Academy of Sciences, 2020. Available from: https://nam.edu/duty-to-plan-health-care-crisis-standards-ofcare-and-novel-coronavirus-sars-cov-2/

5. AESKU.Group [Internet]. 6122 SARS-CoV-2 NP IgG. Accessed: 28 Sep 2020. Available from: https://www.aesku. com/index.php/diagnostics/aeskulisa-elisa-test-kits/infectiousserology-elisa/item/398

6. Silveira MF, Barros AJ, Horta BL, et al. Population-based surveys of antibodies against SARS-CoV-2 in Southern Brazil. Nat Med 2020;26:1196-9.

7. Nisar MI, Ansari N, Amin M, et al. Serial population based serosurvey of antibodies to SARS-CoV-2 in a low and high transmission area of Karachi, Pakistan. medRxiv 2020.

8. Roche Diagnostics [Internet]. Elecsys ${ }^{\circledR}$ Anti-SARS-CoV-2. 2020. Accessed: 28 Sep 2020. Available from: https://diagnostics.roche.com/global/en/products/params/elecsys-anti-sarscov-2.html

9. Stites EC, Wilen CB. The interpretation of SARS-CoV-2 diagnostic tests. Med (N Y) 2020;1:78-89.

10. Hou H, Wang T, Zhang B, et al. Detection of IgM and IgG antibodies in patients with coronavirus disease 2019. Clin Trans1 Immunology 2020;9:e01136.

11. Liu X, Wang J, Xu X, et al. Patterns of IgG and IgM antibody response in COVID-19 patients. Emerg Microbes Infect 2020;9:1269-74.

12. Lippi G, Salvagno GL, Pegoraro M, et al. Preliminary evaluation of Roche Cobas Elecsys Anti-SARS-CoV-2 chemiluminescence immunoassay. Clin Chem Lab Med 2020;58:e251-3. 